\title{
Einstein-Smoluchowsky equation handled by complex fractional moments
}

\author{
Gioacchino Alotta, Mario Di Paola \\ Dipartimento di Ingegneria Civile, Ambientale, Aerospaziale, dei Materiali (DICAM), \\ Universitá degli Studi di Palermo, Viale delle Scienze Ed. 8, 90128, Palermo, Italy, Email: gioacchino.alotta@unipa.it
}

\begin{abstract}
In this paper the response of a non linear half oscillator driven by $\alpha$-stable white noise in terms of probability density function (PDF) is investigated. The evolution of the PDF of such a system is ruled by the so called Einstein-Smoluchowsky equation involving, in the diffusive term, the Riesz fractional derivative. The solution is obtained by the use of complex fractional moments of the PDF, calculated with the aid of Mellin transform operator. It is shown that solution can be found for various values of stability index $\alpha$ and for any nonlinear function of the drift term in the stochastic differential equation.
\end{abstract}

Keywords- $\alpha$-stable white noise, Nonlinear systems, EinsteinSmoluchowsky equation, Complex fractional moments

\section{INTRODUCTION}

The generalization of the Brownian motion $B(t)$ is the $\alpha$ stable Lévy motion $L_{\alpha}(t)(0<\alpha \leq 2)$ [1]. For $\alpha=2, L_{\alpha}(t)$ reverts to the Brownian motion $B(t) . L_{\alpha}(t)$ and $B(t)$ experience some common features: i) Both have independent and orthogonal stationary increments with $L_{\alpha}(0)=0, B(0)=0$; ii) Both have continuous trajectories nowhere differentiable on time. The main difference are: i) $B(t)$ is Gaussian while the Lévy motion $L_{\alpha}(t)$ is not; ii) The mean square of $L_{\alpha}(t)$ no longer grows linearly in time as the Brownian motion. The smaller $\alpha$ the greater the departure from Gaussianity is so generating a large variety of superdiffusive motion.

The (formal) derivative of the Lévy motion gives the $\alpha$-stable white noise. For Langevin differential equations enforced by normal white noise (formal derivative of the Brownian motion) the probability density function is ruled by the well known Fokker-Planck-Kolmogorov (FPK) equation [2]. If the input is the $\alpha$-stable white noise then the PDF of the response process is ruled by the so called Einstein-Smoluchowsky (ES) equation [3]. The aforementioned equations differ each another in the diffusive term. In the former case the diffusive term is the second derivative of the PDF of the response while in the latter the diffusive term is the Riesz fractional derivative of order $\alpha$ of the PDF. Since for $\alpha=2$ the Riesz fractional derivative coalesces with the second derivative, then it may be concluded that the E-S equation is the generalization of the FPK equation. The fractional derivative reflects the nonlocal character in space of the diffusive term.

Various method for finding approximate solution of the FPK equation have been proposed in literature including path integral solution [4]-[8], Wiener Path [9], stochastic averaging method [10], [11],finite element method [12], [13]. Comparatively few papers have been devoted to the solution of the ES equation see e.g. [3], [4], [14]-[21]. And in these papers the PDF of the response is given in exact form only for steady state solution and for particular classes of nonlinearities.
Recently the second author proposed an approximate method for finding the solution of the FPK equation in terms of Complex Fractional Moments (CFM) [22] that are moments of the type $E\left[|X|^{\gamma-1}\right], \gamma \in \mathbb{C}$. These complex quantities are related to the Mellin transform of the PDF [23]-[25]. The appealing in using such quantities instead integer or fractional moments with real exponent relies in the following points: i) moments of the type $E\left[|X|^{\gamma-1}\right]$ never diverge provided the real parts of $\gamma$ belongs to the fundamental strip of the Mellin transform even for $\alpha$-stable processes; ii) both the PDF and Characteristic function are fully restored in the respective domains by inverse Mellin Transform theorem. Because of these remarkable properties in this paper the solution of the ES equation in terms of complex fractional moments is proposed. The method is available for any nonlinear function of the drift term in the stochastic differential equation as well as for non stationary input-output response.

\section{MELLIN TRANSFORM AND COMPLEX FRACTIONAL MOMENTS}

Let $p_{X}(x, t)$ be the probability density function of the random process $X(t)$. Let us now suppose that $p_{X}(x, t)$ is a symmetric distribution, that is $p_{X}(x, t)=p_{X}(-x, t) \forall t$.

The Mellin transform of $p_{X}(x, t)$ is:

$$
\mathscr{M}\left\{p_{X}(x, t) ; \gamma\right\}=\int_{0}^{\infty} p_{X}(x, t) x^{\gamma-1} d x ; \quad \gamma=\rho+I \eta
$$

In the following we denote the Mellin Transform of $p_{X}(x)$ as $M_{p}(\gamma-1, t)$. The inverse Mellin transform returns $p_{X}(x, t)$ in the form

$$
p_{X}(x, t)=\frac{1}{2 \pi i} \int_{\rho-i \infty}^{\rho+i \infty} M_{p}(\gamma-1) x^{-\gamma} d \gamma ; x>0
$$

It is to be emphasized that the integration is performed along to the imaginary axis while $\rho$ remain fixed. The condition for the existence of Eqs (1) and (2) depends on the trend of $p_{X}(x, t)$ at $x=0$ and $x=\infty$. This condition is guarrantee if $-p<\rho<-q$ where $p$ and $q$ are the order of zero at $x=0$ and $x=\infty$ on the $p_{X}(x, t)$. Such an example if $p_{X}(x, t)$ is an $\alpha$-stable distribution, then since the decay of the PDF is for $x$ very large in the form $\tilde{x}^{-\alpha-1}(x>0)$, the order of zero at infinity is $-q=\alpha+1$, in zero the PDF is in general different from zero $\left(\operatorname{order} x^{0}\right.$ ) then for an $\alpha$-stable distribution the existence condition of Eqs (1) and (2) is $0<\rho<\alpha+1$. 
The admissible values for $\rho$, namely $-p<\rho<-q$ is the so called fundamental strip of the Mellin transform. Inspection of Eqs (1) and (2) reveals that whatever the value of $\rho$ is chosen, provided it belongs to the fundamental strip, the value of $p_{X}(x, t)$ is fully restored in the whole range.

Discretization of Eq. (2) gives

$$
\begin{aligned}
& p_{X}(x)=\frac{\Delta \eta}{2 \pi} \sum_{k=-m}^{m} M_{p}\left(\gamma_{k}-1\right) x^{-\gamma_{k}} ; \quad x>0 ; \\
& \gamma_{k}=\rho+i k \Delta \eta
\end{aligned}
$$

where $\Delta \eta$ is the discretization of the $\eta$ axis and $m \Delta \eta$ is a cut off value $\bar{\eta}=m \Delta \eta$ chosen in such a way that terms of higher order than $m \Delta \eta$ do not produce sensible variations on $p_{X}(x, t)$.

In the following we define CFM quantities of the type $E\left[|X|^{\gamma-1}\right]$. By definition

$$
\int_{-\infty}^{\infty} p_{X}(x, t)|x|^{\gamma-1} d x=E\left[|X|^{\gamma-1}\right]
$$

and because of the definition in Eq. (1), in the case of symmetric distribution, $E\left[|X|^{\gamma-1}\right]$ is related to the Mellin transform as

$$
M_{p}(\gamma-1)=\frac{1}{2} E\left[|X|^{\gamma-1}\right]
$$

By inserting this relation into Eq. (3) we recognize the $p_{X}(x, t)$ is returned by the Complex Fractional Moments (CFM) of the PDF.

Some very useful properties of the Mellin transform may be easily demonstrated: i) The CFM are complex functions and are holomorph into the fundamental strip; ii) $E\left[|X|^{\rho+i \eta-1}\right]=E\left[|X|^{\rho+i \eta-1}\right]^{*}$ where the star means complex conjugate; iii) Every distribution possess a finite fundamental strip since $p_{X}(x)$ is positive and its total area is finite (unitary area); iv) Because of the property i) as $\eta \rightarrow \infty$, $E\left[|X|^{\rho+i \eta-1}\right] \rightarrow 0$; v) The result of Eq. (3) is independent of the value of $\rho$ selected, provided it belongs to the fundamental strip.

\section{EINSTEIN SMOLUCHOWSKY EQUATION}

Let the nonlinear Langevin equation enforced by the $\alpha$ stable white noise $W_{\alpha}(t)$ be given in the form

$$
\left\{\begin{array}{l}
\dot{X}=f(X, t)+W_{\alpha}(t) \\
X(0)=X_{0}
\end{array}\right.
$$

where $f(X, t)$ is any nonlinear function of the response process $X(t)$ and $X_{0}$ is a random variable with assigned probability density function. For simplicity sake's we assume that $f(X, t)=-f(-X, t), p_{X}(x, 0)=p_{X_{0}}(x)$ is a symmetric PDF and $W_{\alpha}$, formal derivative of the Lévy $\alpha$-stable process $L_{\alpha}(t)$, has a symmetric distribution with these assumptions
$p_{X}(x, t)=p_{X}(-x, t) \forall t$. The more general case of non symmetric distribution may be treated in a similar way [25].

The Itô equation associated to Eq. (6a) may be written in the form

$$
d X(t)=f(X, t) d t+d L_{\alpha}(t)
$$

where the characteristic function $(\mathrm{CF})$ of $d L_{\alpha}(t)$ (for symmetric $\alpha$-stable process) is in the form

$$
\phi_{d L_{\alpha}}(\theta)=\exp \left(-d t \sigma|\theta|^{\alpha}\right)
$$

where $\sigma$ is the scale factor and $\alpha$ is the stability index. The equation ruling the evolution of the PDF of the response process $X(t)$, namely the ES equation, is given in the form

$$
\frac{\partial p_{X}(x, t)}{\partial t}=-\frac{\partial}{\partial x}\left(f(x, t) p_{X}(x, t)\right)+\sigma^{\alpha} D_{x}^{\alpha}\left(p_{X}(x, t)\right)
$$

where the symbol $D_{x}^{\alpha}(\cdot)$ is the Riesz fractional derivative defined as

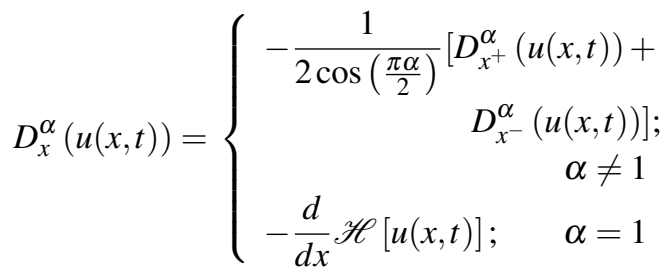

where $D_{x^{+}}^{\alpha}$ and $D_{x^{-}}^{\alpha}$ are the left and right hand side Liouville-Weyl derivative given as

$$
\begin{aligned}
& D_{x^{+}}^{\alpha}(u(x, t))=\frac{1}{\Gamma(n-\alpha)} \frac{d^{n}}{d x^{n}} \int_{-\infty}^{x} \frac{u(\xi, t)}{(x-\xi)^{\alpha-n-1}} d \xi \\
& D_{x^{-}}^{\alpha}(u(x, t))=\frac{1}{\Gamma(n-\alpha)}\left(-\frac{d}{d x}\right)^{n} \int_{-\infty}^{x} \frac{u(\xi, t)}{(\xi-x)^{\alpha-n-1}} d \xi
\end{aligned}
$$

where $\Gamma(\cdot)$ is the Euler Gamma function and the symbol $[\alpha]$ denotes the integer part of the real number $\alpha$. It follows that for $0<\alpha<1$ then $n=1$, while $1 \leq \alpha<2 n=2$. In Eq. (10b) the symbol $\mathscr{H}[\cdot]$ is the Hilbert transform operator

$$
\mathscr{H}[u(x, t)]=\frac{1}{\pi} \mathscr{P} \int_{-\infty}^{\infty} \frac{u(\xi, t)}{x-\xi} d \xi
$$

and $\mathscr{P}$ stands for principal value.

It may be demonstrated that the remarkable property

$$
\mathscr{F}\left\{D_{x}^{\alpha}(u(x, t)), \theta\right\}=-|\theta|^{\alpha} \mathscr{F}\{u(x, t), \theta\}
$$

holds true, where $\mathscr{F}\{u(x, t), \theta\}$ is the Fourier transform operator 


$$
\mathscr{F}\{u(x, t), \theta\}=\int_{-\infty}^{\infty} e^{i \theta x} u(x, t) d x
$$

Because of the property expressed in Eq. (13), the spectral counterpart of the Eq.(9), namely the equation ruling the evolution of the characteristic function $\phi_{X}(\theta, t)$ is given as

$$
\left\{\begin{array}{l}
\frac{\partial \phi_{X}(\theta, t)}{\partial t}=i \theta E[f(X, t) \exp (i \theta X)]- \\
\phi_{X}(\theta, 0)=\phi_{X_{0}}(\theta)
\end{array}\right.
$$

where $\phi_{X_{0}}(\theta)$ is the $\mathrm{Cf}$ at $t=0$.

If the nonlinear function $f(x, t)$ belongs to the general class

$$
f(x, t)=-\sum_{j=0}^{r} c_{j}|x|^{\beta_{j}} \operatorname{Sgn}(x)
$$

then Eq. (15a) is given in the form

$$
\frac{\partial \phi_{X}(\theta, t)}{\partial t}=\theta \sum_{j=0}^{r} c_{j} \tilde{D}_{\theta}^{\beta_{j}} \phi_{X}(\theta, t)-\sigma^{\alpha}|\theta|^{\alpha} \phi_{X}(\theta, t)
$$

where $\tilde{D}_{\theta}^{\beta_{j}}(\cdot)$ is the complementary Riesz fractional derivative defined as

$$
\tilde{D}_{\theta}^{\beta_{j}} \phi_{X}(\theta, t)=\left\{\begin{array}{rr}
-\frac{1}{2 \sin \left(\frac{\pi \beta_{j}}{2}\right)}\left[D_{\theta^{+}}^{\beta_{j}}\left(\phi_{X}(\theta, t)\right)-\right. \\
\left.D_{\theta^{-}}^{\beta_{j}}\left(\phi_{X}(\theta, t)\right)\right] \\
\beta_{j} \neq 1,3, . . \\
i^{\beta_{j} \frac{\partial^{\beta_{j}}}{\partial \theta^{\beta_{j}}} \phi_{X}(\theta, t) ;} \quad \beta_{j}=1,3, . .
\end{array}\right.
$$

The inverse Fourier transform of the complementary Riesz fractional derivative is

$$
\left\{\begin{array}{c}
\mathscr{F}^{-1}\left\{D_{\theta}^{\beta_{j}} \phi_{X}(\theta, t) ; x\right\}=-i \operatorname{Sgn}(x)|x|^{\beta_{j}} p_{X}(x, t) ; \\
\beta_{j} \neq 1,3, . . \\
\mathscr{F}^{-1}\left\{i^{\beta_{j}} \frac{\partial^{\beta_{j}}}{\partial \theta^{\beta_{j}}} \phi_{X}(\theta, t) ; x\right\}=-x^{\beta_{j}} p_{x}(x, t) ; \\
\beta_{j}=1,3, . .
\end{array}\right.
$$

\section{SOlution OF EINSTEIN SMOLUCHOWSKY EQUATION IN TERMS OF CFM}

In this section solution in terms of CFM for the ES equation is pursued.

Let the Eq. (9) particularized for the nonlinearity expressed in Eq. (16), that is

$$
\begin{array}{r}
\frac{\partial p_{x}(x, t)}{\partial t}=\sum_{j=1}^{r} c_{j} \frac{\partial}{\partial x}\left(|x|^{\beta_{j}} \operatorname{Sgn}(x) p_{X}(x, t)\right)+ \\
\sigma^{\alpha} D_{x}^{\alpha}\left(p_{X}(x, t)\right)
\end{array}
$$

The Mellin transform of this equation is

$$
\begin{aligned}
\frac{\partial M_{p}(\gamma-1, t)}{\partial t}=\sum_{j=1}^{r} c_{j}\left[x^{\gamma-1+\beta_{j}} p_{X}(x, t)\right]_{0}^{\infty}- \\
(\gamma-1) \sum_{j=1}^{r} c_{j} M_{p_{X}}\left(\gamma-2+\beta_{j}, t\right)- \\
\sigma^{\alpha} \frac{v_{c}(\gamma)}{v_{c}(\gamma-\alpha)} M_{p}(\gamma-1-\alpha, t)
\end{aligned}
$$

where $v_{c}(\gamma)=\Gamma(\gamma) \cos (\pi / 2 \gamma)$. The first term of right hand side (r.h.s.) of Eq. (21) is zero if $\rho-1+\beta_{j}>0$, moreover if $\lim _{x \rightarrow \infty} p_{X}(x, t) \sim x^{-u}$ then the second limitation is $\rho-1+\beta_{j}-$ $u<0$. If these limitations are respected Eq. (21) becomes:

$$
\begin{aligned}
& \frac{\partial M_{p}(\gamma-1, t)}{\partial t}=-(\gamma-1) \sum_{j=1}^{r} c_{j} M_{p}\left(\gamma-2+\beta_{j}, t\right)- \\
& \sigma^{\alpha} \frac{v_{c}(\gamma)}{v_{c}(\gamma-\alpha)} M_{p}(\gamma-1-\alpha)
\end{aligned}
$$

This equation may be particularized for different values of $\gamma$, say $\gamma_{k}=\rho+i k \Delta \eta(k=-m, . ., 0, . . m)$ obtaining a set of $2 m+$ 1 ordinary linear differential equations. At this point it is not possible to solve such a set of differential equations because the fractional moments are evaluated for different value of $\rho$. This problem is analogue to that of the infinite hierarchy. To solve this problem we observe that Eq. (3) can be written for different values of $\rho$, provided it belongs to the fundamental strip; so we can equate Eq. (3) particularized for two different values of $\rho, \rho_{1}=\rho$ and $\rho_{2}=\rho+\Delta \rho$ and we indicate with $M_{p}\left(\gamma_{k}^{(1)}-1, t\right), M_{p}\left(\gamma_{k}^{(2)}-1, t\right)$ the CFM evaluated in $\gamma_{k}^{(j)}=$ $\rho_{j}+i k \Delta \eta$, with $j=1,2$ :

$$
\begin{aligned}
x^{-\rho_{1}} \frac{\Delta \eta}{2 \pi} \sum_{s=-m}^{m} M_{p}\left(\gamma_{s}^{(1)}-1, t\right) x^{-i s \frac{\pi}{b}}= \\
x^{-\rho_{2}} \frac{\Delta \eta}{2 \pi} \sum_{k=-m}^{m} M_{p}\left(\gamma_{k}^{(2)}-1, t\right) x^{-i k \frac{\pi}{b}}
\end{aligned}
$$

where $b=\pi / \Delta \eta$. By multiplying both side of this equation for $x^{-1 / 2}$ we can write: 


$$
\begin{aligned}
x^{-1 / 2} & \sum_{s=-m}^{m} M_{p}\left(\gamma_{s}^{(1)}-1, t\right) e^{-i s \frac{\pi}{b} \ln x}= \\
& x^{-(\Delta \rho+1 / 2)} \sum_{k=-m}^{m} M_{p}\left(\gamma_{k}^{(2)}-1, t\right) e^{-i k \frac{\pi}{b} \ln x} \quad x>0 ;
\end{aligned}
$$

It is to be remarked that Eq. (24) strictly holds for $x>0$ because in zero singularities appears. Now we suppose $M_{p}\left(\gamma_{k}^{(2)}-\right.$ $1, t)$ is known and we want to evaluate $M_{p}\left(\gamma_{k}^{(1)}-1, t\right)$. Because Eq. (3) is an approximation, we require that Eq. (24) holds true in a weak sense in the interval $x_{1}>0, x_{2} \gg 0$ :

$$
\begin{array}{r}
\int_{x_{1}}^{x_{2}} \frac{1}{x}\left\{\left[\sum_{s=-m}^{m} M_{p}\left(\gamma_{s}^{(1)}-1, t\right) e^{-i s \frac{\pi}{b} \ln x}-\right.\right. \\
\left.x^{-\Delta \rho} \sum_{k=-m}^{m} M_{p}\left(\gamma_{k}^{(2)}-1, t\right) e^{-i k \frac{\pi}{b} \ln x}\right] \times \\
[\text { C.C. }]\} d x=\min \left(M_{p}\left(\gamma_{k}^{(1)}-1, t\right)\right)
\end{array}
$$

where $[$ C.C. $]$ stands for complex conjugate. Now we make a change of variables by putting

$$
\xi=\ln x, d \xi=\frac{d x}{x} ; \xi_{j}=\ln x_{j}, j=1,2
$$

In order to find $M_{p}\left(\gamma_{k}^{(1)}, t\right)$ as a linear combination of $M_{p}\left(\gamma_{s}^{(1)}, t\right)$ we perform variations and we put $x_{1}=e^{-b}, x_{2}=e^{b}$ instead of $x_{1}=0, x_{2}=\infty$, that may not be enforced since in 0 singularities appear. With these positions two goals are achieved: i) since $b=\pi / \Delta \eta$, and $\Delta \eta$ is of order $0.1 \div 0.5$, the interval $e^{-b} \div e^{b}$ is very large, that is we require that $p_{X}(x, t)$ will match the effective PDF in a wide range but the singularities in 0 are excluded; ii) with this choice, after substituting Eqs. (26) in Eq. (25), the integral is in the range $-b \div b$ and before the variations is given as

$$
\begin{array}{r}
\int_{-b}^{b}\left\{\left[\sum_{s=-m}^{m} M_{p}\left(\gamma_{s}^{(1)}-1, t\right) e^{-i s \frac{\pi}{b} \ln x}-\right.\right. \\
\left.e^{-\Delta \rho \xi} \sum_{k=-m}^{m} M_{p}\left(\gamma_{k}^{(2)}-1, t\right) e^{-i k \frac{\pi}{b} \ln x}\right] \times \\
[\text { C.C. }]\} d x=\min \left(M_{p}\left(\gamma_{s}^{(1)}-1, t\right)\right)
\end{array}
$$

In the range $e^{-b} \div e^{b}$ we can take advantage of the orthogonality condition of $e^{-i k \frac{\pi}{b} \xi}$ in $-b \div b$ so that after minimization we obtain

$$
2 b M_{p}\left(\gamma_{s}^{(1)}-1, t\right)=\sum_{k=-m}^{m} M_{p}\left(\gamma_{k}^{(2)}-1, t\right) a_{k s}(\Delta \rho)
$$

where

$$
\begin{aligned}
& a_{k s}(\Delta \rho)=\int_{-b}^{b} e^{-\Delta \rho \xi} e^{-i(k-s) \frac{\pi}{b}} d \xi= \\
& \frac{2 b \sin [\pi(k-s)-i b \Delta \rho]}{\pi(k-s)-i b \Delta \rho}
\end{aligned}
$$

With the aid of Eq. (28) we can obtain $M_{p}\left(\gamma_{s}^{(1)}-1, t\right)$ as a linear combination of $M_{p}\left(\gamma_{k}^{(2)}-1, t\right)$ and solve differential equations by using Mellin transform. With these results we may solve also E-S equation. Eq.(22) require $M_{p}\left(\gamma_{k}-1, t\right)$, $M_{p}\left(\gamma_{k}-2+\beta_{j}, t\right)$ and $M_{p}\left(\gamma_{k}-1-\alpha, t\right)$; we select a proper initial value of $\rho$ in order to fulfill the limitations $\rho-1+\beta_{j}>$ $0, \rho-1+\beta_{j}-u<0$ and the general limitation $\rho<1+\alpha$ and we evaluate

$$
\begin{aligned}
& M_{p}\left(\gamma_{s}+\beta_{j}-2, t\right)=\frac{1}{2 b} \sum_{k=-m}^{m} M_{p}\left(\gamma_{k}-1, t\right) a_{k s}(\Delta \rho) \\
& M_{p}\left(\gamma_{s}-1-\alpha, t\right)=\frac{1}{2 b} \sum_{k=-m}^{m} M_{p}\left(\gamma_{k}-1, t\right) a_{k s}(\alpha)
\end{aligned}
$$

By inserting these equations in Eq. (22) particularized for $\gamma=\gamma_{s}$, we get a set of $2 m+1$ complex ordinary differential equations in the unknown $M_{p}\left(\gamma_{s}-1, t\right)$. To find the correct solution of such as system it is necessary to impose that the area under the PDF in the interval $e^{-b} \div e^{b}$ is $1 / 2$. This condition may be enforced very easily, taking into account Eq. (3), as follows

$$
\frac{1}{2 b} \sum_{s=-m}^{m} M_{p}\left(\gamma_{s}-1, t\right) \int_{e^{-b}}^{e^{b}} x^{-\gamma_{s}} d x=\frac{1}{2}
$$

From this equation we get

$$
M_{p}\left(\gamma_{0}-1, t\right)=b-\sum_{s=-m}^{m} M_{p}\left(\gamma_{s}-1, t\right) ; \quad s \neq 0
$$

By inserting this condition into Eq. (22) and inserting Eqs. (30) particularized for $s=-m, . .,-1,1, \ldots, m$ we get a set of $2 m+1$ differential equations ruling the evolution of the CFM. After the solution of such a system the PDF at any instant $t$ can be reconstructed thanks to Eq. (3). In the next section some applications are presented for various values of $\alpha$ and $\beta$.

\section{NUMERICAL APPLICATION}

In this section we show the solution of the ES equation for linear $(\beta=1)$ and non-linear $(\beta=0,0.3,0.5,1.5,3)$ half oscillator forced by $\alpha$-stable white noise with various value of stability index $(\alpha=2,1)$. The case $\alpha=2$ correspond to the Fokker-Planck equation yet solved with the same method in [22]. In all the examples reported the oscillator is quiescent for $t<0$, so the PDF is a Dirac Delta and the CFMs are all zero; at 


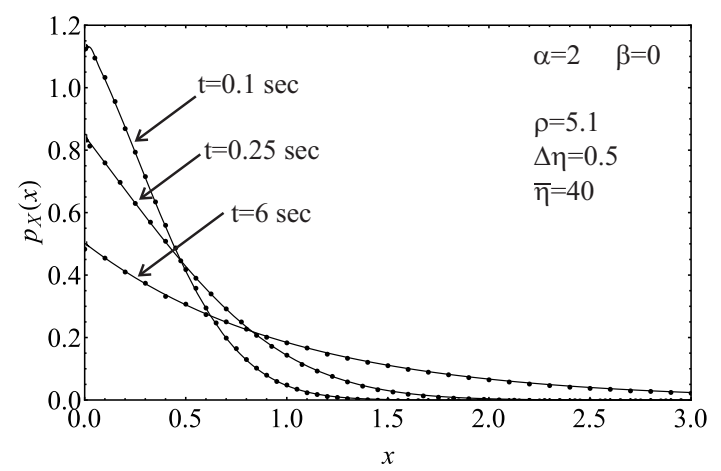

(a)

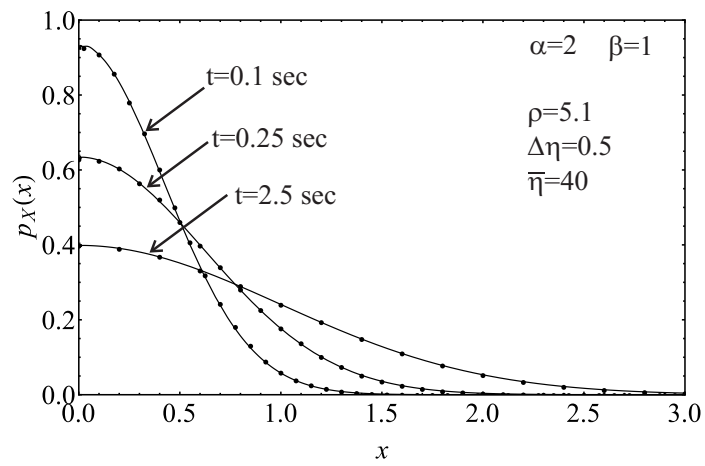

(c)

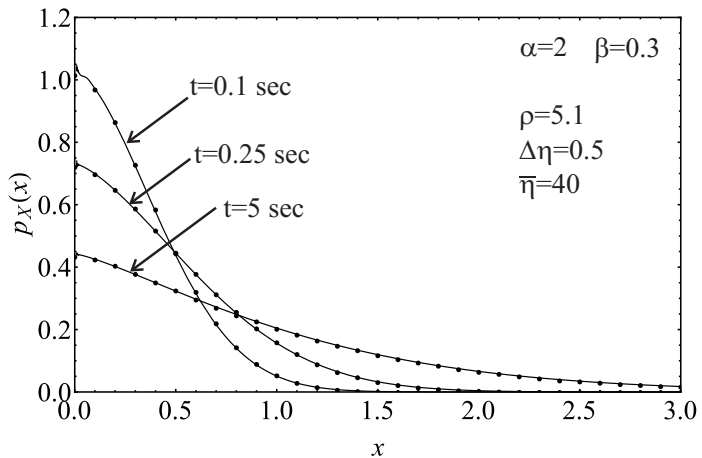

(b)

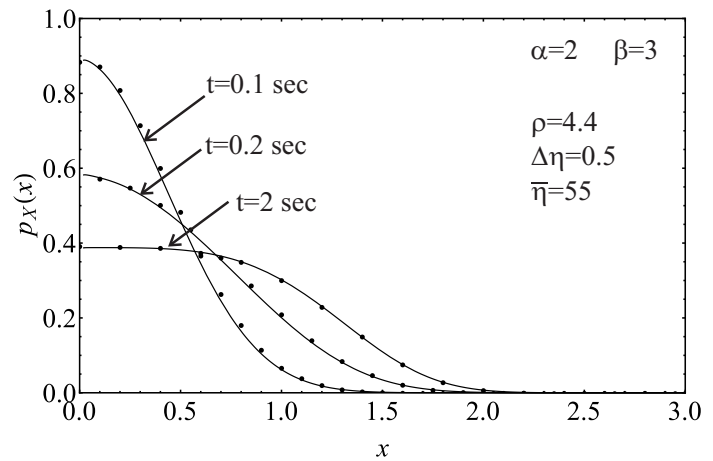

(d)

Figure 1. CFM solution (continuous line) versus PDF by digital simulation (dotted curves) for $\alpha=2$ at various instant and for different $\beta$.

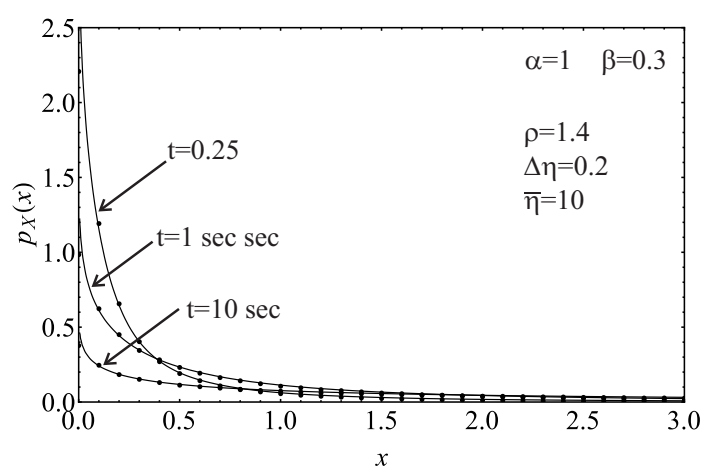

(a)

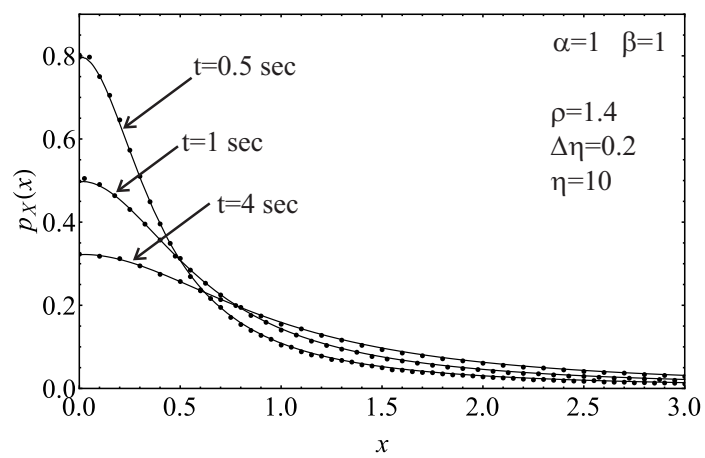

(c)

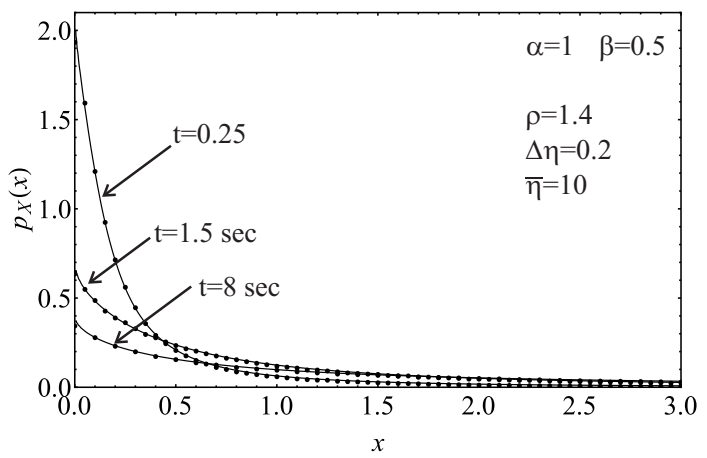

(b)

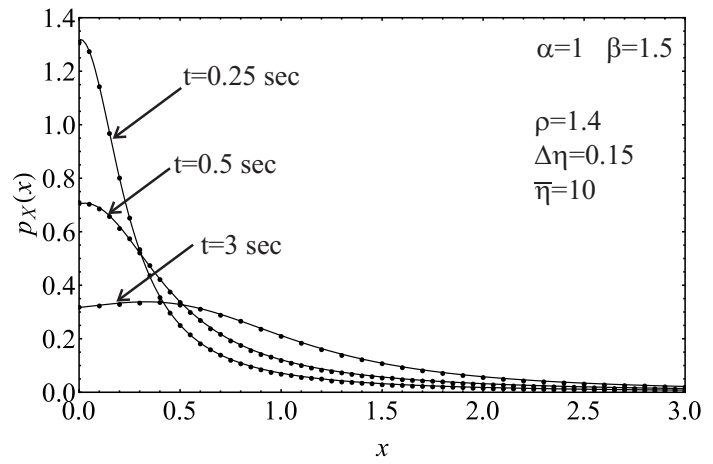

(d)

Figure 2. CFM solution (continuous line) versus PDF by digital simulation (dotted curves) for $\alpha=1$ at various instant and for different $\beta$. 
$t=0$ we apply an $\alpha$-stable white noise with scale factor $\sigma=1$, skewness $\beta=0$, shift $\mu=0$. The solution obtained with the proposed method is contrasted with the PDF constructed by digital simulations with $10^{6}$ samples.

\section{A. $\alpha=2$}

This value of $\alpha$ correspond to the case of the the FokkerPlanck equation, in which the input reverts to the normal white noise. In this case the fundamental strip is $0<\rho<\infty$ and this allow us to use Eq. (30a) for any value of $\beta$ and so for any value of $\Delta \rho$. This means that we can find solutions for any nonlinear $f(X, t)$ in Eq. (6a).

\section{B. $\alpha=1$}

This case is taken as general case in the range $0 \leq \alpha \leq 2$; the PDF of the input is the well known Cauchy distribution. When stability index is lesser than 2 the fundamental strip depend on the values of $\alpha$ and $\beta$, because of the decay of the PDF for $x \rightarrow \infty$. In particular in [3] it has been demonstrated that for $\alpha$-stable input, the tails of the PDF decay as a power law $x^{-u}$, being $u=\alpha+1$ for the linear half-oscillator $(\beta=1)$ and $u=\alpha+3$ for the quartic oscillator $(\beta=3)$, so in both cases $u=\alpha+\beta$. By substituting this equality in the condition $\rho-1+\beta-u<0$ we obtain $\rho<1+\alpha$, so we can say that the fundamental strip for $\alpha$-stable input is always $0 \div 1+\alpha$. This is a limitation that actually can't be eliminated, so we can study systems where the parameters are such that always exist all Mellin transform of Eq.(22). Because of this limitation in Fig. 2 the maximum value of $\beta$ is 1.5 .

\section{CONCLUSION}

In this paper the response in terms of PDF of a nonlinear system forced by an external $\alpha$-stable white noise is studied. The governing equations of such a system in terms of PDF is the so called Einstein-Smoluchowsky equation obtained with the aid of Itó calculus. The solution of these equation is found by the Mellin transform operator that permit us to transform the terms of the E-S equation in complex fractional moments of the PDF; these moments are finite only if the Mellin transform is performed with a value of $\rho$ belonging to the fundamental strip of the PDF. The key of the method is to write the CFMs calculated for different value of $\rho$ as a linear combination of the CFMs of an arbitrary value of $\rho$; once this aim is obtained the E-S equation is transformed into a set of complex ordinary differential equations that can be easily solved with a symbolic or numeric package. The numerical applications show the accuracy of the prediction of the PDF in any instant of the temporal range at hands. Lastly, it is important to underline that this method can be easily extended to systems forced by asymmetric $\alpha$-stable white noise and to more complex systems also involving fractional terms.

\section{REFERENCES}

[1] G. Samorodnitsky, M. S. Taqqu. Stable non-Gaussian random processes Stochastic models with infinite variance, Chapman \& Hall: London, 1994.

[2] H. Risken. The Fokker Planck equation, Springer: Berlin, 1996.

[3] A. Chechkin, A. V. Gonchar, J. Klafter, R. Metzeler, L. Tanatorov. Stationary states of non-linear oscillators driven by Lévy white noise, Chemical Physics, vol. 284, pp. 233-251, 2002.
[4] F. Mainardi, Y. Luchko, G. Pagnini. The fundamental solution of the space-time fractional diffusion equation, Fractional calculus and applied analysis, vol. 4, pp. 153-192, 2001.

[5] R. Iwankiewicz, S.R.K. Nielsen. Solution techniques for pulse problems in non-linear stochastic dynamics Original Research Article, Probabilistic Engineering Mechanics, vol. 15, pp. 25-36, 2000.

[6] A. Naess, V. Moe. Efficient path integration methods for nonlinear dynamic systems, Probabilistic Engineering Mechanics, vol. 15, pp. 221231, 2000 .

[7] E. Mamontov, A. Naess. An analytical-numerical method for fast evaluation of probability densities for transient solutions of nonlinear Itô's stochastic differential equations, International Journal Engineering Dynamics, vol. 47, pp. 116-130, 2009.

[8] A. Pirrotta, R. Santoro. Probabilistic response of nonlinear systems under combined normal and Poisson white noise via path integral method, Probabilistic Engineering Mechanics, vol. 25, pp. 25-32, 2011.

[9] I.A. Kougioumtzoglou, P.D. Spanos. An analytical Wiener path integral technique for non-stationary response determination of nonlinear oscillators, Probabilistic Engineering Mechanics, vol. 28, pp. 125-131, 2012.

[10] W.Q. Zhu. Stochastic averaging method in random vibration, Applied Mechanics Reviews, vol. 41, pp. 189-199, 1988.

[11] J.B. Roberts, P.D. Spanos. Stochastic averaging: An approximate method of solving random vibration problems Review Article, International Journal Engineering Dynamics, vol. 21, pp. 111-134, 1986.

[12] L.A. Bergman, B.F. Spencer. On the numerical solution of the FokkerPlanck equation for nonlinear stochastic systems, Nonlinear Dynamics, vol. 4, pp. 357-372, 1993.

[13] G. Stefanou. The stochastic finite element method: Past, present and future Review Article, Nonlinear Dynamics, vol. 4, pp. 357-372, 2009.

[14] M. Di Paola, G. Failla. Stochastic response analysis of linear and nonlinear systems to $\alpha$-stable Lévy white noise, Probabilistic Engineering Mechanics, vol. 20, pp. 128-135, 2005.

[15] M. Di Paola, A. Pirrotta, M. Zingales. Itô calculus extended to systems driven by $\alpha$-stable Lévy white noises (a novel clip on the tails of Lévy motion), International Journal Engineering Dynamics, vol. 42, pp. 10461054, 2007.

[16] B. Dybiec, I.M. Sokolov, A. V. Chechkin. Stationary states in single-well potentials under symmetric Lévy noise, Journal of Statistical Mechanics: Theory and Experiment, vol. 7, P07008, 2010.

[17] V. Yu. Gonchar, L. V. Tanatarov, A. V. Chechkin. Stationary solutions of the fractional kinetic equation with a symmetric power-law potential, Theoretical and Mathematical Physics, vol. 131 (1), pp. 582-594, 2002.

[18] M. Grigoriu. Equivalent linearization for systems driven by Lévy white noise, Probabilistic Engineering Mechanics, vol. 15, pp. 185-190, 2000.

[19] M. Grigoriu. Characteristic function equation for the state of dynamic system with Gaussian, Poisson and Lévy white noise, Probabilistic Engineering Mechanics, vol. 19, pp. 449-461, 2004.

[20] G. Samorodnitsky, M. Grigoriu. Characteristic function for the stationary state of a one dimensional dynamical system with Lévy noise, Theoretical and Mathematical Physics, vol. 150, pp. 332-346, 2007.

[21] G. Cottone. Statistics of nonlinear dynamical systems under Lévy noises by a convolution quadrature approach, Journal of Physics A: Mathematical and Theoretical, vol. 44 (18), 185001, 2011.

[22] M. Di Paola. Fokker Planck equation solved in terms of complex fractional moments, Probabilistic Engineering Mechanics, (in print).

[23] G. Cottone, M. Di Paola. On the use of fractional calculus for the probabilistic characterization of random variable, Probabilistic Engineering Mechanics, vol. 24, pp. 321-330, 2009.

[24] G. Cottone, M. Di Paola, R. Metzler. Fractional calculus approach to the statistical characterization of random variables and vectors, Physica A: Statistical Mechanics and its Applications , vol. 389, pp. 909-920, 2010.

[25] M. Di Paola, F.P. Pinnola. Riesz fractional integrals and complex fractional moments for the probabilistic characterization of random variables, Probabilistic Engineering Mechanics, vol. 29, pp. 149-156, 2012. 